\title{
Towards the Maturation of IT Usability Evaluation (MAUSE)
}

\author{
Effie L.-C. Law ${ }^{1}$, Ebba T. Hvannberg ${ }^{2}$, Gilbert Cockton ${ }^{3}$, Philippe Palanque ${ }^{4}$, \\ Dominque Scapin ${ }^{5}$, Mark Springett ${ }^{6}$, Christian Stary ${ }^{7}$, and Jean Vanderdonckt ${ }^{8}$ \\ ${ }^{1}$ ETH Zürich, Switzerland ${ }^{2}$ University of Iceland, UK ${ }^{3}$ University of Sunderland, UK \\ ${ }^{4}$ Universite Paul Sabatier, France ${ }^{5}$ INRIA Rocquencourt, France ${ }^{6}$ University of Middlesex, \\ UK ${ }^{7}$ University of Linz, Austria ${ }^{8}$ Catholic University of Louvain, Belgium
}

\begin{abstract}
This article describes a new initiative MAUSE of which the ultimate goal is to bring more science to bear on usability evaluation methods. This overarching goal will be realized through scientific activities of four Working Groups (WGs) with each of them having specific objectives, rationales, tasks and expected outcomes. Outlook for MAUSE's development is described.
\end{abstract}

\section{Introduction}

MAUSE (Towards the MAturation of Information Technology USability Evaluation), COST294, is a recent initiative with the mission of bringing more science to bear on the development, evaluation and comparison of Usability Evaluation Methods (UEMs), aiming to drive this young and significant Research \& Development area towards maturity and to yield results that can be transferred to industry and educators.

A UEM is defined as any systematic method or technique employed to perform usability evaluation of any interactive design at any stage of its development. The objective of usability evaluation is to determine whether a system fulfils a set of usability requirements. The output is a set of deviations from the requirements, which may be a list of usability problems (UPs), expressed as undesirable design features, scenarios or improvement suggestions, but these deviations may also be described with other measures that indicate problematic usages. Usability evaluation research has been poorly focused and the findings are inconclusive. Consequently, many serious UPs still occur in all kinds of software products. This fact also suggests that the integration of software design and usability evaluation in industrial software development has largely been unsuccessful. Indeed, there are a number of definitional, theoretical and methodological issues in usability research. Furthermore, the emerging landscape of Information Technologies (IT) such as Ambient Intelligence and Pervasive Computing calls for the adaptation as well as extension of existing UEMs. Basic and intricate problems in usability research need to be resolved by extensive co-operation among a community of usability professionals and researchers with diversified backgrounds. MAUSE-COST294 is a structured fouryear action being operated under the auspices of the European Science Foundation to orchestrate collaborative efforts of usability experts from a number of European countries (http://www.cost294.org). The action was launched in January 2005. 


\section{Objectives and Four Working Groups}

The main objective of MAUSE is threefold:

- To deepen understanding about the inherent strengths and weaknesses of individual Usability Evaluation Methods (UEMs);

- To identify reliable and valid methods for comparing different UEMs in terms of their effectiveness, efficiency and scope of applicability;

- To develop effective strategies for extracting useful information from the results of UEMs in order to improve the systems tested

MAUSE comprises four WGs with each of them having specific objectives, rationales, tasks and expected outcomes. They are interdependent to a certain extent; results from one can facilitate the progress of the others and vice-versa.

\section{WG1: Critical Review and Analysis of Individual UEMs}

Objective: To build a refined, substantiated and consolidated knowledge-pool about usability evaluation based on the expertise, experiences and research work of the participating partners.

Rationale: The fundamental problem is that most UEMs are not strongly rooted in a clearly defined and sound theoretical framework [8], but are rather pragmatic. Further, no effective strategies are yet available to manage (never mind eradicate) systematically the user/evaluator effect [5], which are known to substantially undermine the reliability and validity of results of UEMs. How variants of think-aloud methods (concurrent vs. retrospective) [9] and sets of usability guidelines (Nielsen vs. Gerhardt-Powals) [6] influence UEM results entail more empirical data.

Tasks: UEMs of interest are critically reviewed and analyzed on various attributes:

- underlying theoretical background - explaining the effect of a UEM

- scope of application - range of conditions and contexts where a UEM is deployed

- cost-effectiveness - person-hours versus effectiveness as specifically defined

- user/evaluator effect - means to measure and mitigate this undesirable effect

- limitations of the techniques - identifying remedial strategies

- level of acceptance - extent of usage in the industry and academic community

- possible extension - increasing scope of application and effectiveness

Outcome: A database is created to record the findings of individual project teams involved in MAUSE with regard to the aforementioned attributes of different UEMs. These data serve as important reference points from which insights into the future development of UEMs can be gained. Best practices of different UEMs, covering operational, organizational as well as cultural dimensions, can be derived from records of this database and rendered accessible to researchers and practitioners.

\section{WG2: Comparing UEMs: Strategies and Implementation}

Objective: To identify effective strategies to compare different UEMs.

Rationale: There is currently poor understanding of the scope of different UEMs in terms of who, when, how and where they are used. UEMs tend to yield different insights into usability issues and these differences are not well understood or 
articulated [cf. 7]. There is a need for well-defined and tested methods for establishing that scope. Further, in the literature [2,3] different comparison criteria have been proposed, but they are loosely defined and thus measured differently.

Tasks: Empirical, analytic and model-based UEMs will be compared. Major tasks are:

- To conduct meta-data analysis of the empirical studies implemented in the context of MAUSE and those available in the literature

- To develop comprehensive, consistent and robust means to compute different comparison criteria, especially thoroughness, validity and reliability

- To implement comparisons among different UEMs or among different usage conditions of a single UEM as it is applied to different software systems. Multisited experiments involving different project teams will be coordinated to carry out both types of comparisons

- To develop quality models for the Web to enable a benchmarking comparing different UEMs

Outcome: Recommendation on the deployment of a particular UEM, under certain conditions and contexts with the expected effectiveness, can be proposed to usability practitioners.

\section{WG3: Refining and Validating Classification Schemes for Usability Problems}

Objective: To improve and substantiate the tools for classifying and analyzing usability problems with the goal of enhancing feedback to design and process improvement

Rationale: There is a lack of a thoroughly validated defect classification scheme (DCS) specifically designed for analyzing usability problems based on the integration of basic concepts of HCI and software engineering. Such a scheme is crucial for identifying origins of usability problems, for extracting useful information to improve the systems evaluated and for facilitating the communication between usability specialists and software developers.

Tasks: (i) To review and compare existing DCSs (e.g. CUP [4], UAF [1]) for their suitability and adaptability for isolating causes of usability problems and for proposing re-design solutions; (ii) To validate CUP with various types of software applications, ranging from mobile PDA to large screen display devices, by evaluators with different expertise and experience; (iii) To explore the possibility of developing a robust DCS by integrating the findings to be obtained in (i) and (ii).

Outcome: Refined and validated DCSs will be disseminated to other usability professionals. Deeper understanding of the origins of UPs can shed some light on the significant issue of integrating usability into the systems development lifecycle.

\section{WG4: Review on Computational and Definitional Approaches in Usability Evaluation}

Objective: To review systematically the existing models and procedures for estimating certain key usability test parameters and the traditionally defined usability quality metrics. Of primary interest are the capabilities of formal representations.

Rationale: Several fundamental definitions and standard approaches entail further systematic investigations and refinements. 
Tasks: The work will be guided by six strategic principles: (i) Computational UEM Relevance - a meta-model for computing indicators and prospective indices for human behaviour and system performance; (ii) Definitional Primacy - a real and broadly accepted UP specification; (iii) Fine-grain UEM Specification - identifying a comprehensive list of UEM attributes; (iv) Baseline Specificity Exploitation addressing user, system and organizational baseline measures; (v) Explication of Semantic Encodings - mapping a usability-parameter definition to a computational scheme; rendering the semantic relationships between the three traditional usability metrics transparent; (vi) UEM Accuracy Determination - the adequacy of the means for capturing interactive software quality

Outcome: With the improved models and algorithms, more accurate estimations of key usability test parameters can be obtained. Practitioners might re-arrange usability evaluation accordingly, based on revised usability metrics, operational criteria for UPs and in line with other quality attributes upheld by other professionals.

\section{Outlook}

MAUSE-COST294 brings together leading HCI researchers from different European countries with each of them being highly knowledgeable in usability evaluation. The concerted efforts being contributed by these experts will lead to fruitful outcomes. Apart from the Working Groups, other instruments for fostering the attainment of MAUSE's overarching goal are Short-Term Scientific Missions and Training Schools for young researchers. Further, given the wide networks that the partners are continuously extending to the academic community, standardization bodies and industry for exchanging knowledge and practice, results from MAUSE-COST294 can be disseminated effectively, thereby enabling their uptake. Since the action is still in its infancy, concrete outcomes with respect to the problems addressed above are not yet available and will become more visible during its course of development.

\section{References}

1. Andre, T.S., Hartson, H.R., Belz, S.M., \& McCreary, F.A. (2001). The user action framework. International Journal Human-Computer Studies, 54: 107-136.

2. Cockton, G., D. Lavery, \& A. Woolrych (2003). Inspection-based evaluation. In J. A. Jacko \& A. Sears (Eds.), Human-Computer Interaction Handbook (pp. 1118-1138). Erlbaum.

3. Hartson, H.R., Andre, T.S., Williges, R.C. (2003). Criteria for evaluating usability evaluation methods. Int. J. Human and Computer Interaction 15(1): 145-181.

4. Hvannberg, E.T., \& Law, E.L.-C. (2003).Classification of Usability Problems (CUP) Scheme. In Proceedings of INTERACT 2003.

5. Law, E. L.-C., \& Hvannberg, E.T. (2004a). Analysis of combinatorial user effect in international usability tests. In Proceedings of CHI 2004.

6. Law, E. L.-C., \& Hvannberg, E.T. (2004b). Analysis of strategies for improving and estimating the effectiveness of heuristic evaluation. In: Proceedings of NordiCHI 2004.

7. Molich R., Ede M., Kaasgaard K., \& Karyukin B.(2004). Comparative usability evaluation. Behaviour and Information Technology, 23(1): 65-74.

8. Rogers, Y. (2004). New theoretical approaches for HCI. Annual Review of Information Science and Technology (ARIST), Volume 38.

9. Van den Haak, M.J., de Jong, M.D.T., Schellens, P. (2003). Retrospective vs. concurrent think-aloud protocols. Behaviour and Information Technology, 22 (5), 339-351. 